\title{
Interface effect of ultrafine mineral particles and microorganisms
}

\author{
Faqin Dong ${ }^{1} \cdot$ Jianxi Zhu ${ }^{2} \cdot$ Siwang $\mathrm{Yu}^{3} \cdot$ Liang Bian $^{1}$ \\ Received: 5 July 2018 / Accepted: 16 July 2018 / Published online: 28 July 2018 \\ (C) Springer-Verlag GmbH Germany, part of Springer Nature 2018
}

Ultrafine mineral particles and individual microorganisms are important components of atmospheric particulate matter and have high correlations in morphology, coexistence, and aerodynamic behavior. However, the interaction between atmospheric particles and microorganisms in air is ignored while much attention has paid to the harm caused by atmospheric particles to humans. In addition to serving as a carrier for microorganisms, a living nutrient source for organisms, and indirectly reducing the atmospheric $\mathrm{CO}_{2}$ content, atmospheric particulate matter may also have impact on microorganisms due to their own size effect, surface activity, and component toxicity, so as to increase the degree of joint harm to humans and expand the transmission route. Interface effect of ultrafine mineral particles and microorganisms has great significance in atmospheric ultrafine mineral microbial biomigration and adsorption, surface biological change behavior, inhalable mineral granules (IMG) hazards, and toxicological risk reasonable assessment. It is essential for developing a scientific air quality standard, origination of air transport fine

Responsible editor: Philippe Garrigues

Faqin Dong

fqdong2004@163.com

Jianxi Zhu

zhujx@gig.ac.cn

Siwang Yu

swang_yu@hsc.pku.edu.cn

Liang Bian

bianliang@swust.edu.cn

1 Key Laboratory of Solid Waste Treatment and Resource Recycle, Ministry of Education, Southwest University of Science and Technology, Mianyang 621010, China

2 Guangzhou Institute of Geochemistry, Chinese Academy of Sciences, Guangzhou 510640, China

3 State Key Laboratory of Natural and Biomimetic Drugs; Department of Molecular and Cellular Pharmacology, Peking University School of Pharmaceutical Sciences, Beijing 100191, China particles and collection of ancient biological environment information, biological metallurgy and soil synergies activation of IMG microbial/gas/solid, even biological protection in aircraft. To verify IMG/single, multi-strain particle interaction mechanism, the change of mineral particle size, surface morphology of IMG in interfacial interaction process, surface charge, surface groups, mineral surface adsorption, and migration and change of elements, phase change, surface restructuring, dissolution, and the type and quantity of free radicals over the time must be considered. Through analyzing adsorption process and mineral crystallization induced by bacteria, exploring the amount and species change of acid, enzymes, sugar, the microbial oxidation damage, and other biological effects in vitro by Ames test et al., studying the variation of cell membrane structure and metabolism of common microorganism, and then proving the microorganisms activity characteristic expression of fine IMG and its attachment, the interface characteristics and the production of biofilm interaction, the toxicity model of IMG interface/microbial membrane system could be established.

After a rigorous peer-review process, 15 papers about mineral particles and microorganisms or cells were accepted for publication in the Environmental Science and Pollution Research Special Issue: IMPM. The brief introduction of articles accepted for SI: IMPM is given as follows:

In the paper of "The interface interactions behavior between E. coli and two kinds of fibrous minerals," batch experiments were performed to deal with the interaction of Escherichia coli and two fibrous minerals (brucite and palygorskite), and the interface and liquid phase characteristics in the short-term interaction processes were discussed. Studies of short-term interaction between $E$. coli and brucite and palygorskite can help to understand the effect of fibrous minerals on microeubiosis of human normal flora and the contribution of microbial behaviors on the fibrous minerals weathering in the natural environment.

$\mathrm{Li}$ et al. found that the existence of the bacteria cells and EPS significantly affected the surface properties of calcite and provides a theoretical basis for the mechanism of PM fine particulate matter on human health impact for further study. 
To explore the process and the results of microbial cells and their extracellular polymeric substances (EPS) acting on the surface properties of minerals of $\mathrm{PM}_{2.5}$ through the metabolism, a common native soil bacterium Bacillus mucitaginosus with abundant extracellular polymers was chosen as the tested strain. Meanwhile, as one of the $\mathrm{PM}_{2.5}$ common minerals, calcite fine particles were taken as the research object to explore the influence of microbial cells and extracellular polymers on its surface properties.

The wide use of asbestos and its substitutes has given rise to studies on their possible harmful effects on human health and environment. However, their toxic effects remain unclear. In the paper titled "In vitro genotoxicity of asbestos substitutes induced by coupled stimulation of dissolved high-valence ions and oxide radicals," Huo et al. aimed to disclose the coupled effects of dissolved high-valence ions and oxide radicals using the in vitro cytotoxicity and genotoxicity of chrysotile (CA), nano- $\mathrm{SiO}_{2}$ (NS), ceramic fiber (CF), glass fiber (GF), and rock wool (RW) on Chinese hamster lung cells V79. In conclusion, ions from short-time dissolution of dust samples and the generation of extracellular $\cdot \mathrm{OH}$ presented combined effects in the elevation of intracellular ROS, which further synergistically induced cytotoxicity and genotoxicity.

In the paper of "Interface effect of natural precipitated dusts on the normal flora of Escherichia coli and Staphylococcus epidermidis," Deng et al. investigated the interface effect the interface effect between five types of natural precipitated dust and two normal floras. Results showed that the concentration of Ca showed a significant increase upon interaction with $E$. coli and S. epidermidis in all dusts. Besides, the results also showed an apparent increase in the concentration of pyruvate, 0205-galactosidase, and alkaline phosphatase (AKP) after being co-cultured with E. coli and S. epidermidis, in which FC-1 \# is enhanced in the most obvious. Plenty of S. epidermidis interacted with dust gathered in the indentations of dust, particularly in pleated surfaces. Further, these findings demonstrated that the alkaline dust with higher $\mathrm{Ca}$ content stimulated the growth of bacteria, and irregularly shaped or thin dust would be easier to combine with bacteria and conduct interface effect.

It was reported that in vitro short-term exposure to $\mathrm{PM}_{2.5}$ caused different lung diseases through inflammatory response, immune toxicity, oxidative stress, and genetic mutations. Yang et al. investigated the cytotoxicity, oxidative damage, mitochondria damage, apoptosis, and cell cycle arrest of $\mathrm{NX}$ and QH $\mathrm{PM}_{2.5}$ in A549 cells. Further, cell cycle arrestrelated gene levels in $\mathrm{PM}_{2.5}$-induced A549 cells were also detected. Results suggested that excessive ROS decreased MMP levels and damaged mitochondrial membrane integrity and induced mitochondrial oxidative damage through the oxygen-dependent killer route, resulting in mitochondrial damage and cell apoptosis. Besides, the results also showed that $\mathrm{PM}_{2.5}$ induced $\mathrm{A} 549$ cell cycle alteration in $\mathrm{G} 2 / \mathrm{M}$ phase after co-culture for $24 \mathrm{~h}$. G2/M phase arrest was induced by upregulation of p53 and p21 and downregulation of CDK1 mRNA expression. In addition, lncRNA Sox 2 ot might play an important role as the specific oncogenes and it participated in $\mathrm{G} 2 / \mathrm{M}$ phase arrest by regulating the expression of $\mathrm{EZH}_{2}$.

Zeng et al. investigated the Lung Injury and expression of p53, p16 of Wistar rats by respirable chrysotile fiber dust in four primary areas of China. They found that the respirable chrysotile fiber dust from the four primary areas of China had the risk of causing lung injury, and these changes may be related to the physical and chemical characteristics of chrysotile from different production areas.

In the paper of "Chrysotile and Rock Wool Fibers Induce Chromosome Aberrations and DNA Damage in V79 Lung Fibroblast Cells," chrysotiles from Mangnai, Qinghai, China, and an artificial substitute, rock wool fiber were prepared as suspensions and were tested at different concentrations in V79 lung fibroblasts. Chromosome aberrations were detected by micronucleus assay after exposure for $24 \mathrm{~h}$, and DNA damage was estimated by single-cell gel electrophoresis after exposure for 12, 24, or $48 \mathrm{~h}$. According to the results, chrysotile and rock wool fibers caused micronuclei to form in a dose-dependent manner in V79 cells; olive tail moment values increased in a dose- and time-dependent manner. When V79 cells were exposed to a concentration of $200 \mu \mathrm{g} /$ $\mathrm{mL}$, the degree of DNA damage induced by chrysotile fibers was greater than rock wool fibers. The study suggests that both chrysotile and rock wool fibers could induce chromosome aberrations and DNA damage.

The carcinogenicity of chrysotile has been extensively documented, and accumulative evidence has shown that chrysotile is capable of causing lung cancer and other forms of cancer. However, molecular mechanisms underlying the tumorigenic effects of chrysotile remained poorly understood. In the paper titled "Chrysotile effects the expression of antioncogene p53 and p16 and oncogene c-jun and c-fos in wistar rats lung tissues," it was found that chrysotile exposure leads the body weight to grow slowly and lung viscera coefficients to increase in a dose-dependent manner. General sample showed white nodules, punctiform asbestos spots, and irregular atrophy; moreover, HE staining revealed inflammatory infiltration, damage of alveolar structures, agglomerations, and pulmonary fibrosis. In addition, chrysotile can induce inactivation of the anti-oncogene P53 and P16 and activation of the proto-oncogenes C-JUN and C-FOS both in the messenger RNA and protein level.

Zhao et al. investigated the variation of preserving organic matter bound in the interlayer space of montmorillonite (Mt) induced by a microbe metabolic process in the paper "Variation of Preserving Organic Matter Bound in Interlayer of Montmorillonite Induced by Microbial Metabolic Process." Results showed that the montmorillonite under the influence of soil bacteria has a strong buffering capacity for preserving 
organic matter into the interlayer space in a short-term. It might provide critical implications for understanding the evolution process and the preservation of fertilization which was in the over-fertilization or less-fertilization conditions on farmland.

Research into the mechanism for interactions between bacterium and radionuclides is the starting point for achieving successful remediation of radionuclides with microorganisms. The paper titled "Synergistic Interface Behavior of Strontium Adsorption Using Mixed Microorganisms" using Sr(II) as a simulation radionuclide and the mixed microorganisms of Saccharomyces cerevisiae and Bacillus subtilis as the biological adsorbent investigated behavior at the interface between $\mathrm{Sr}$ (II) and the microorganisms as well as the mechanisms governing that behavior. The results showed that $\mathrm{Sr}$ (II) biosorption onto the mixed microorganisms is spontaneous and endothermic in nature. The key interaction between the biological adsorbent and $\mathrm{Sr}$ (II) involves shared electronic pairs arising from chemical reactions via bond complexation or electronic exchange, and spectral and energy spectrum analyses show that functional groups at the interface between the radionuclide and the mixed microorganisms are the main active sites of the interface reactions.

Photoelectrochemical process is an environmentally friendly technology and has a wide application in the control of environmental pollutants. Efficient nanophotocatalysts responsive to visible light are still highly attractive. In the paper titled "Improving photoelectrochemical reduction of $\mathrm{Cr}$ (VI) ions by building $\alpha-\mathrm{Fe}_{2} \mathrm{O}_{3} / \mathrm{TiO}_{2}$ electrode," $\alpha-\mathrm{Fe}_{2} \mathrm{O}_{3} / \mathrm{TiO}_{2}$ were grown on fluorine-doped tin oxide (FTO) substrates by hydrothermal method for photoelectrochemical reduction of $\mathrm{Cr}(\mathrm{VI})$. Compared with the separate $\alpha-\mathrm{Fe}_{2} \mathrm{O}_{3}$ and $\mathrm{TiO}_{2}$ electrodes, the composite $\alpha-\mathrm{Fe}_{2} \mathrm{O}_{3} / \mathrm{TiO}_{2}$ electrodes showed higher photocurrent density. Under visible light irradiation, $100 \%$ removal efficiency of $\mathrm{Cr}(\mathrm{VI})$ was obtained after 40 min treatment. The composite $\alpha-\mathrm{Fe}_{2} \mathrm{O}_{3} / \mathrm{TiO}_{2}$ electrodes showed an enhanced absorbance in visible light region and had good stability to photoelectrochemical reduction of $\mathrm{Cr}(\mathrm{VI})$.

In the paper of "Adsorption and mineralization of rare earth elements onto bacterial cell surface," Cheng et al. explored the use of Bacillus licheniformis to adsorb lanthanum and subsequent mineralization process in contaminated water. The maximum adsorption capacity of lanthanum on bacteria was $113.98 \mathrm{mg} / \mathrm{g}$ (dry weight) biomass. X-ray diffraction (XRD) and transmission electron microscopy (TEM) data indicated that adsorbed lanthanum on bacterial cell surface occurred in an amorphous form at the initial stage. Scanning electron microscopy with X-ray energy-dispersive spectroscopy (SEM/ EDS) results indicated that lanthanum adsorption was correlated with phosphate. The amorphous material was converted into scorpion-like monazite ( $\mathrm{LaPO}_{4}$ nanoparticles) in a month.

Cigarette smoke causes insulin resistance, which is associated with type 2 diabetes mellitus (T2DM). Because the involvement of microRNAs (miRNAs) in the development of insulin resistance is largely unknown, Yang et al. investigated, in hepatocytes, the roles of miR-191 in cigarette smoke extract (CSE)-induced insulin resistance. In L-02 cells, CSE not only decreased glucose uptake and glycogen levels but also reduced levels of insulin receptor substrate-1 (IRS-1) and Akt activation, effects that were blocked by SC79, an activator of Akt. CSE also increased miR-191 levels in L-02 cells. Furthermore, the inhibition of miR-191 blocked the decreases of IRS-1 and p-Akt levels, which antagonized the decreases of glucose uptake and glycogen levels in L-02 cells induced by CSE. These results reveal a mechanism by which miR-191 is involved in CSE-induced hepatic insulin resistance via the IRS-1/Akt signaling pathway, which helps to elucidate the mechanism for cigarette smoke-induced T2DM.

In the paper of "Application of protein typing in molecular epidemiological investigation of nosocomial infections outbreak of aminoglycoside-resistant Pseudomonas aeruginosa," the authors conducted to understand the prevalence of aminoglycoside (AMG)-resistant $P$. aeruginosa in our hospital and to provide a scientific basis for control measures against nosocomial infections. SELDI-TOF MS technology can be used for bacterial typing, which provides a new method of clinical epidemiological survey and nosocomial infection control.

In the study of "A synergetic immobilization strategy for removing strontium during calcification of the coccolithophore Emiliania huxleyi," stable strontium immobilization during the calcification process was investigated to indirectly assess a proposed bioremediation approach for removing $\mathrm{Sr}^{2+}$ contamination from marine environments. Results indicate that E. huxleyi has high $\mathrm{Sr}^{2+}$ tolerance and removal efficiency in response to $\mathrm{Sr}^{2+}$ stress ranging from 5.6 to $105.6 \mathrm{ppm}$. $\mathrm{Sr}^{2+}$ immobilization during E. huxleyi calcification indicates a concentration-dependent synergistic mechanism. At lower concentrations of $\mathrm{Sr}^{2+}$ (25.6 ppm), $\mathrm{Sr}^{2+}$ is incorporated into coccoliths through competitive supply between $\mathrm{Sr}^{2+}$ and $\mathrm{Ca}^{2+}$. In addition, calcite productivity decreases with increased $\mathrm{Sr}^{2+}$ removal efficiency due to crystallographic transformation of coccoliths from hydrated calcite into aragonite at $55.6 \mathrm{ppm} \mathrm{Sr}^{2+}$. Further formation of strontianite at $105.6 \mathrm{ppm} \mathrm{Sr}^{2+}$ is due to precipitation of $\mathrm{Sr}^{2+}$ on the edge of the rims and radial arrays of the coccoliths. The study implies that coccolithophores are capable of significant removal of $\mathrm{Sr}^{2+}$ from the marine environment.

According to the differentiation problem of the environmental behavior and biological effects in the action process between the inhalable mineral granules and common bacteria and cells, we selected the IMG and common bacteria and cells as the research object that there are high correlation on the morphology, coexistence relationship, and aerodynamic behavior between them and investigated the environmental behavior characteristics of IMG, the free radical characterization and biological activity in the IMG/microbe system, the 
process of the physicochemical reactions, and membrane biological chemistry on the regional surface in the interface of IMG and microbial membrane (interface/membrane interaction). The major mineral phase of IMG in China is quartz, calcite, albite, mica, and montmorillonite, presenting obvious difference that quartz is the main mineral phase in the northern China which the content occupies over $51 \%$ and calcite in the southern districts. Minerals play a key role in the free radical releasing of the IMG in liquid, showing the effect of dose and time. Adhesion of heavy metal $\left(\mathrm{Pb}^{2+}, \mathrm{Cd}^{2+}\right)$, erosion of organic/inorganic acid, and treatment of salt $\left(\left(\mathrm{NH}_{4}\right)_{2} \mathrm{SO}_{4}\right.$, $\mathrm{NH}_{4} \mathrm{Cl}$ ) could disturb the releasing of free radicals. The targets of free radicals focused on the membrane protein and lipids in the bio-membrane causing the oxidative damage of bacteria. Amino acid molecules adsorbed on the surface of IMG by cation exchange and end surface adsorption. The effect of IMG caused the $\beta$-sheets' and $\alpha$-helices' structural change of the protein and phospholipid molecules. The bonding sites of the IMG interacted with E. coli and S. epidermis focused on the phospholipid diester groups of the membrane and amide groups of protein respectively. The bonding way acting on the microbial cell membrane varied with IMG when IMG coated heavy metal. The results of the computational simulation showed that biomolecules, such as phospholipids, protein, and so on, could adsorb on the surface of montmorillonite and quartz by non-valence bonding interactions, and

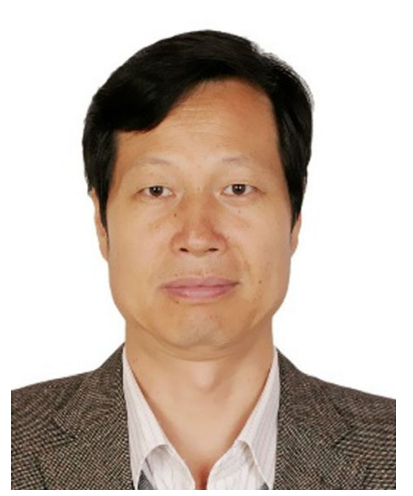

Prof. Faqin Dong, professor/ $\mathrm{PhD}$ supervisor, director of key laboratory of solid waste treatment and resource recycle, ministry of education, vice-president of Southwest University of Science and Technology. Science and technology progress award of Sichuan province, secondclass award of national excellent teaching achievements.

Served as former chairman of international applied mineral committee and is the editorial board member of Acta Mineralogica Sinica and journal of functional materials. The major research fields include mineral materials, ecology, and environment materials. Is responsible for more than 20 projects published above 100 papers, 4 academic books, and 12 approved patents. transferring electrons with atoms $(\mathrm{Al}, \mathrm{O}$, etc.) on mineral surface affected the torsional force of phospholipid and then destroyed its structure. IMG and polluted IMG caused the reactive oxygen (ROS) content of receptor cells increase, the metabolism of energy source (LDH, ATP, ALP, SDH) and redox equilibrium of the bacteria abnormal. However, the IMG-coated heavy metal by different ways induced the antagonism or synergism toxic effect as the difference of variation element, function dose. The group of low dose appeared the effect of toxicity excitement. Extracellular products of common bacteria promote the dissolution, structure collapse, and crystal type transformation of IMG and participate the $\mathrm{Ca}^{2+}$ recrystallization. The influence of organic acid that change the structure and morphology of IMG is particularly significant. The findings provide data support for the environmental safety evaluation of IMG, planning the hygiene standards and environmental standards of fine particulate, mineral modification by microbe and biological metallurgy, having important scientific significance.

This special issue cannot fully reflect the diversity and creativity of the ideas and new insights. However, as editors, we hope that this issue may prompt scientists from diverse fields to pay more attention to the interface effect of mineral particles and microorganisms in atmospheric pollution.

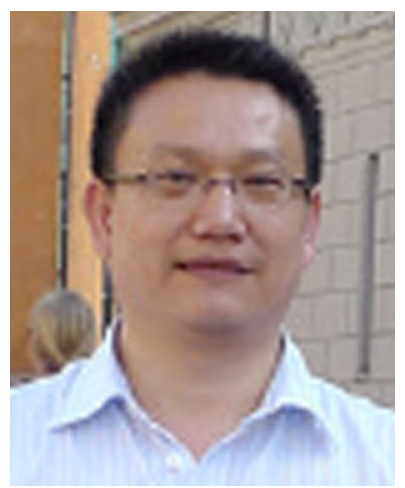

Prof. Jianxi Zhu, Professor/ $\mathrm{PhD}$ supervisor, Director of Guangdong Province Key Laboratory of Mineral Physics and Materials. The winner of Hou-Defeng Award, issued by the society of Mineralogy, Petrology and Geochemistry of China. Serves as the associate editor of Clays and Clay minerals, the editorial board member of Minerals, and Scientific Reports. The major research fields include the surface physical chemistry of minerals and clay mineral-based nanomaterials. Published 150 peer-reviewed international journal articles. 


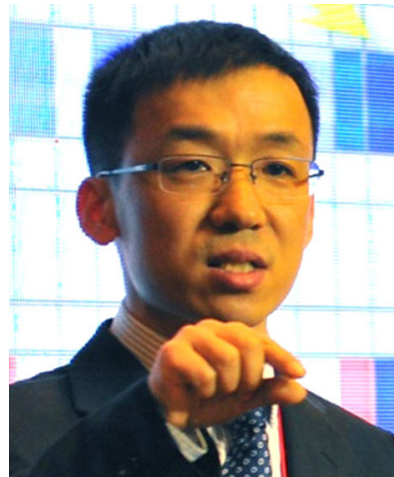

Dr. Siwang Yu is currently an associate professor and Ph.D. supervisor at the Department of Molecular and Cellular Pharmacology, Peking University School of Pharmaceutical Sciences. He serves as an editorial board member of Current Pharmacology Reports and Journal of Chinese Pharmaceutical Sciences and is also a member of Tumor Prevention and Control Committee of Chinese Association of Preventive Medicine, as well as member of American Association of Cancer Research, Chinese Pharmacological Society, Chinese Anti-Cancer Association, and Chinese Chemical Society. The research interests of Dr. Yu focus on the roles of stress responsive signaling in regulation of cellular energetic/xenobiotic metabolism and microenvironment and their impact on cancer initiation, progression, metastasis, drug resistance, and toxicities/adverse effects of chemotherapies, and their potentials as predictive biomarkers and preventive drug targets. Dr. Yu has been the PI of 3 NSFC projects and 1 special 863 innovative project, and actively participated in several NSFC and MoST projects. He has published over 70 scientific articles including more than 50 SCI papers, 5 book chapters, and more than 20 academic conference abstracts/reports.

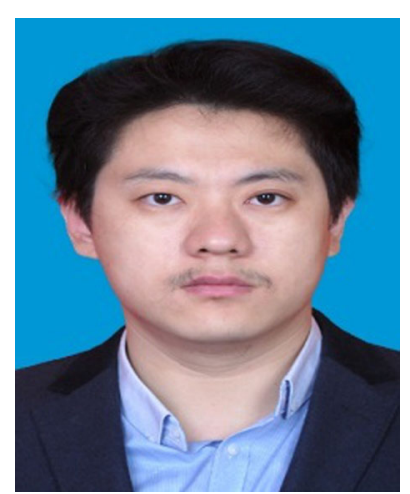

Prof. Liang Bian, $\mathrm{PhD} /$ Professor, winner of the OneThousand-Talents Scheme in Sichuan Province, Hebei outstanding young scholars, Xinjiang science fund of outstanding young scholars. Serves as the member of committee on mineral composites, China composite materials society. The major research fields include the environmental mineralogy and computational mineralogy. Published above 70 SCI papers and 22 approved patents. Is responsible for 14 projects, and got the excellent award of west light foundation of the Chinese Academy of Sciences. 